\title{
Clinical retention force development of double crowns
}

\author{
Stefan Bayer • Helmut Stark • Lina Gölz • \\ Ludger Keilig • Dominik Kraus • Anja Hansen • \\ Norbert Enkling
}

Received: 2 August 2010 / Accepted: 16 February 2011 / Published online: 1 March 2011

(C) Springer-Verlag 2011

\begin{abstract}
This study deals with the development of the retentive forces of double crowns intraorally measured. Twenty-five combined fixed-removable prostheses with a total of 84 double crowns were included in the study. The intraoral measurement was performed at 72 defined measuring points directly adjacent to the double crowns of the dentures. The measurement was performed 4-6 weeks (baseline), 6 months (recall 1), and 18 months (recall 2) after the insertion of the restoration. A specifically designed measuring device was used. The median values for the single measuring points reached $4.705 \mathrm{~N}$ at the baseline, $5.190 \mathrm{~N}$ after 6 months, and $3.740 \mathrm{~N}$ after 18 months. The measured values were analyzed according to differences between the median retention forces at the three defined points in time. The statistical analysis of the median values showed no statistical difference for the retention force change after 6 months but for the decrease until the second recall (Mann-Whitney test). The retention force per denture was calculated by a summation of
\end{abstract}

\footnotetext{
S. Bayer $(\bowtie) \cdot$ H. Stark $\cdot$ D. Kraus $\cdot$ A. Hansen $\cdot$ N. Enkling Department of Prosthodontics, Preclinical Education and Dental Materials Science, Welschnonnenstr.17,

53111 Bonn, Germany

e-mail: sbayer@uni-bonn.de

S. Bayer $\cdot$ N. Enkling

Clinic for Prosthetic Dentistry, University of Bern,

Bern, Switzerland

L. Gölz

Clinic for Orthodontics, University of Bonn,

Bonn, Germany

L. Keilig

Endowed Chair of Oral Technology,

Rheinische Friedrich-Wilhelms University,

Bonn, Germany
}

the single measuring points. At the baseline, $12.9 \mathrm{~N}$ was reached. The forces did only decrease slightly and were not statistically significant. The results indicate that retention force values of double crowns, measured intraorally at the patient, do not relevantly change clinically within the first 1.5 years. Within the limitations of this study, it can be stated that wear does not influence the retentive forces of double crowns within the first 18 months. After this period the retention force should be still sufficient for denture retention.

Keywords In vivo $\cdot$ Clinical retention force $\cdot$ Retention force change $\cdot$ Double crown $\cdot$ Denture $\cdot$ Friction

\section{Introduction}

For the purpose of oral rehabilitation of the partially edentulous dentition, different attachment systems can be used for the retention of combined fixed-partial dentures [1-5]. Telescopic crowns [6-9] as well as conical crowns $[6,10-12]$ are universally established and widely spread for this indication. The recent literature describes the high degree of intraoral comfort and a good long-term viability provided by conical $[10,12,13]$ and telescopic systems $[5$, $8,14]$. A frequent problem of the principle of double crown retention is the frictional wear during the functional period $[8,15]$. Clinical research of this frictional wear requires an intraoral measurement of the retention force change. The examination of the retentive force development could provide indicators for improving this retentive system. As reported by Becker for the telescopic system [16] and Körber for the conical double crowns [17], retention forces of 3.5-7 N per attachment should achieve adequate denture retention. Lehmann and Armin as well as Botega et al. confirmed that these forces were adequate also for bar and 
ball attachments [18, 19]. Current data from measurements performed at clinically used denture showed mean retentive forces of $2 \mathrm{~N}$ [20] to 3-6.5 N [4] for telescopic crowns prior to cementation. For the intraoral measurements of double crowns, current data are missing. Some studies measured the intraoral retention forces of implant-retained dentures. They showed forces about 3-8 $\mathrm{N}$ for magnets, $6-30 \mathrm{~N}$ for ball anchors, and $15-30 \mathrm{~N}$ for bars [21-23]. The values referred to the retention of the denture, not to the single retentive element. All systems showed a retention force decrease after a longer period of time [21].

\section{Materials and methods}

Within the scope of a controlled clinical trial, 23 partially edentulous patients were included in this study. All subjects gave informed consent. The ethics committee of the University of Bonn, Germany approved the study.

The patients received combined fixed-removable partial dentures retained by double crowns. Fifteen dentures were inserted in upper and 10 in lower jaws. Three double crowns were used as median number of attachments per denture (maximum five, minimum one). Normally, the dentures were constructed using a vestibular open design. If this was not esthetically acceptable, an overdenture design with a labial flange was chosen.

The intraoral measurements were performed 4-6 weeks (baseline), 6 months (recall 1), and 18 months (recall 2) after the insertion of the restoration.

Four commercial dental laboratories fabricated the dentures for the operators and student courses of the Department of Prosthodontics, Preclinical Education and Dental Materials Science. The double crowns were fabricated from high-gold-content type 4 alloys. They were not produced as theoretical ideal telescopic crowns with a preparation angle of $0^{\circ}$ but were modified by the dental laboratories by a slight conical angle of $1-2^{\circ}$.

Measuring stylus for the in vitro and in vivo measurement

The retention force was measured using a device specifically designed for the purpose. The measuring device comprised a measuring stylus with a strain gauge (Fig. 1), and the measurements were processed in a PC using an A-D converter card. The resolution of the system was $0.01 \mathrm{~N}$. The retention forces were recorded and analyzed with Dasylab ${ }^{\circledR} 7.0$ (National Instruments).

\section{Measurement procedure}

Seventy-two measuring points were used for the retention force measurements of the inserted dentures. Their position

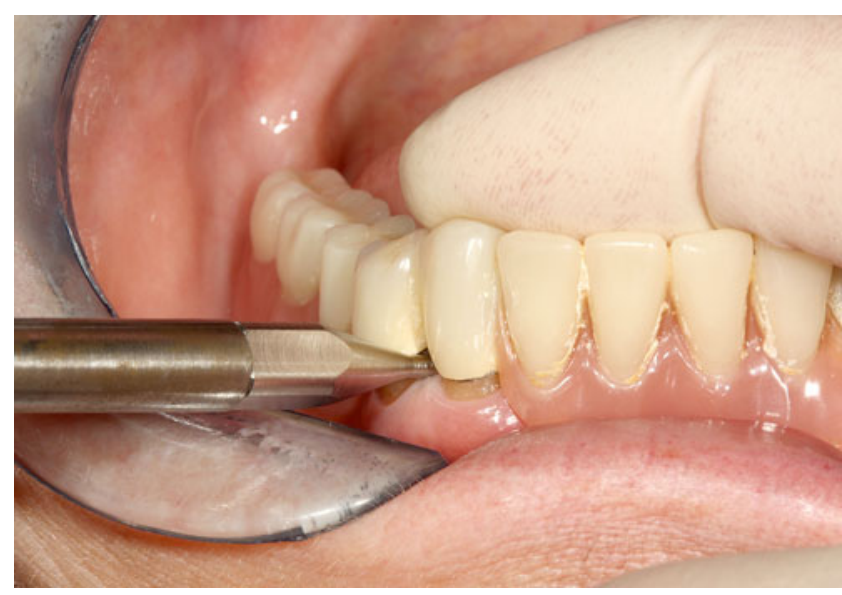

Fig. 1 In vivo measurement. Intraoral retention force measurement at a denture of the lower jaw. Double crowns are found at the left canine and first premolar. The denture is secured by the examiner not to fall of suddenly

was defined and they were used again for every repeated measurement (Fig. 1). Six repeated measurements were accomplished for each measuring point, and the median value was used for further calculation. Before measurement the double crowns were cleansed of adherent food or plaque.

The position of the measuring points was defined by the following concept: (1) The point was defined not to be located further posterior than the distal surface of the second premolars. (2) If there was an adjacent abutment tooth found, the measuring device was positioned between the two teeth. (3) If the tooth had no directly adjacent tooth, the mesial connection to the saddle was used as working point. (4) If a prosthesis using an overdenture design was tested, the measuring point was set at the labial denture flange labial of the abutment tooth. The retentive force was measured for the initial separation of the double crown approximately at the first $0.1 \mathrm{~mm}$ of separation.

\section{Statistical analysis}

For the comparison of the median retention force values, the Mann-Whitney test was used. To get a better comparability with the results of the recent literature, the sum of retention force per denture was calculated. The statistical analysis was performed using Prism 4.0 (Graph Pad Software Inc., La Jolla, CA, USA).

\section{Results}

Retention force of the single measuring points

The results of the retention force measurement are shown in Fig. 2. The retention force at the baseline ranges from 0.65 up to $36.48 \mathrm{~N}$ with a median of $4.705 \mathrm{~N}$ and an interquartile 


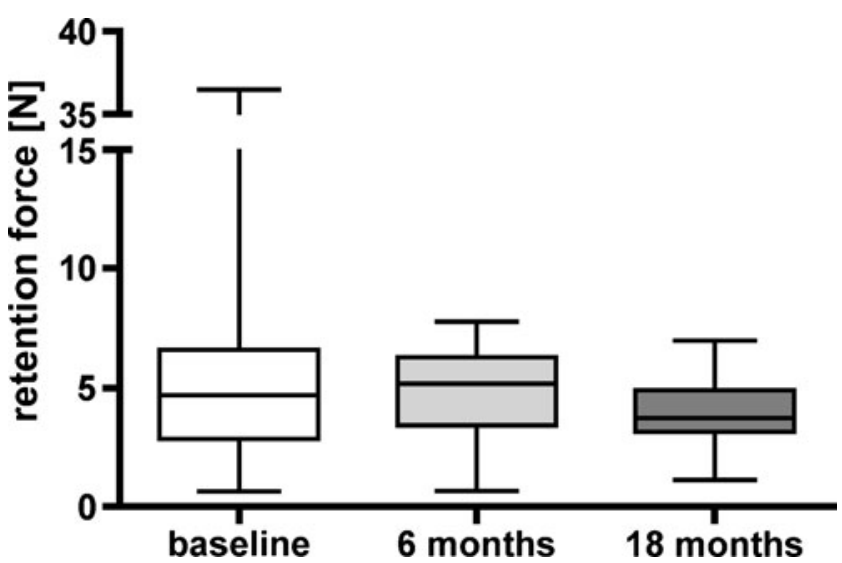

Fig. 2 Diagram of the values of the single measuring points. Comparison of the retention force at the baseline, at 6 (recall 1) and at 18 (recall 2) months after insertion of the denture. The comparison of the values showed a statistical significant difference between the baseline vs. 6-month recall $(p=0.0386)$ and baseline vs. 18-month recall $(p=0.0469)$

range of $3.75 \mathrm{~N}$. The measurements after 6 months reached a slightly higher median force of $5.19 \mathrm{~N}$ with an interquartile range of $2.88 \mathrm{~N}$. The second recall after 18 months showed a decreased median retention force of $3.74 \mathrm{~N}$ with an interquartile range of $1.75 \mathrm{~N}$. The minimum values at the recalls reached about the same dimension as in the baseline measurement (recall $1=0.67 \mathrm{~N}$, recall $2=$ $1.15 \mathrm{~N}$ ). The maximum values reached about one fifth of the maximum value at the baseline (recall $1=7.81 \mathrm{~N}$, recall $2=7.01 \mathrm{~N})$.

The comparison of the median values of all three groups was performed by the Mann-Whitney test. This test showed that the retention force after 18 months was significantly lower than at the baseline $(p=0.0469)$ and the first recall $(p=0.0386)$. The initial increase after 6 months was not statistically significant.

\section{Retention force per denture}

The calculation of the retention force per denture was performed only as summation of the values of the single measurement points. The results of the calculation are shown in Fig. 3. Three double crowns were found as median number of attachments per denture. The retention force at the baseline ranged from 4.0 up to $62.3 \mathrm{~N}$ with a median of $12.9 \mathrm{~N}$ and an interquartile range of $13.4 \mathrm{~N}$. The measurements after 6 months reached a lower median force of $10.4 \mathrm{~N}$ with an interquartile range of $9.4 \mathrm{~N}$. The second recall after 18 months showed a median retention force of $11.1 \mathrm{~N}$ with an interquartile range of $7.7 \mathrm{~N}$.

The comparison of the median values of all three groups was performed by the Mann-Whitney test. This test showed that the retention force of the dentures did not change significantly by the examination period of 18 months.

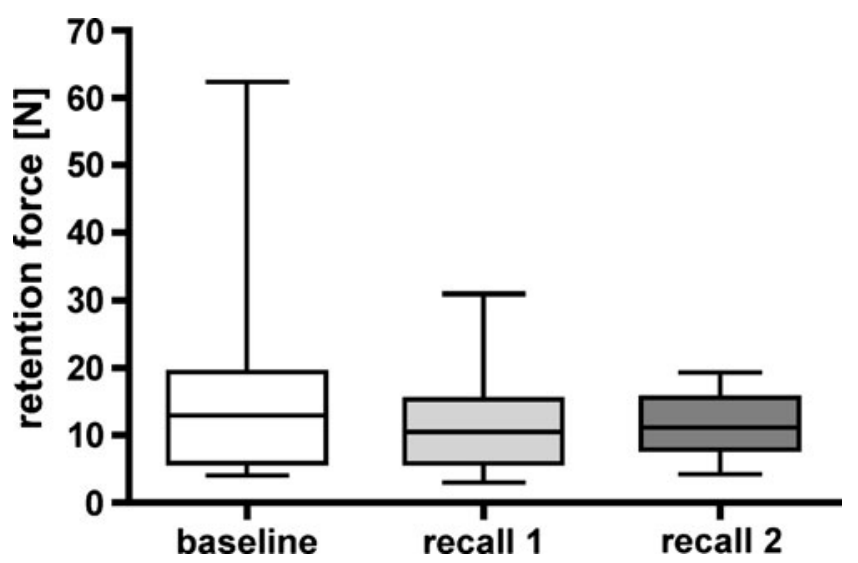

Fig. 3 Diagram of the values calculated per denture. Comparison of the retention force at the baseline, at 6 (recall 1) and at 18 (recall 2) months after insertion of the denture. The comparison of the values showed no statistical significant differences between the three points in time

\section{Discussion}

The cardinal aim of this study was to investigate the clinical development of the retention force values of double crowns. The retention force was determined by an intraoral measurement. For the estimation of the retention force change during a period of 1.5 years, measurements at the baseline, after 6 and 18 months were taken. For a better comparability with the data of the recent literature, a calculation of the retention force per denture was performed.

Method of the measurement setup and procedure

The main problem of the intraoral retention force measurement is the reproducibility of the denture's removal process. All studies with the topic of intraoral retention force measurement have to face this problem. Some experimental setups tried to solve this problem using a fixation of the patient's head combined with a denture removal by a linearmotor-controlled measuring device [22]. This should guarantee the identity of the longitudinal axis of the double crown and the direction of removal. These systems are linked rigidly to the prosthesis. They are used to measure the retention of the whole denture. The problem of such an apparatus is that a perfect reproducibility of the patient's head is not possible. This leads to a divergence between the axes of removal and of the double crown. So, only retentive elements can be examined which do not need an absolutely parallelism of these two axes (ball attachments or magnets). The system is not useful for the retention force measurement of double crown retained dentures as a tilting of the denture could pretend excessive forces not clinically existing. As alternative setup measurement devices linked below, the labial flange of the denture is used [21, 23, 24]. 
These systems are not rigidly connected to the denture. They can be used to measure the retention of a different number of attachments located in the anterior part of the jaw, as the working point for posterior located attachments is unwieldy. A comparable system was used in the present study as it also makes use of a localized working point. This copies the clinical situation of removal forces, caused by sticky food chewed on one side of the denture, in a more realistic way. In this clinical situation, the initial retentive force of the double crown is determining for the dentures stability. Concerning the procedure of retention force measurement, it is important to remove the denture only initially and not as much as possible. Such a further onesided removal causes a tilting of the double crown and thus pretends higher forces than in real terms.

The measuring stylus was orientated in a way that its longitudinal axis built a right angle with the longitudinal axis of the double crown. The denture was secured against a fast and uncontrolled removal by the examiner's fingers. It was necessary to be aware of avoiding a contact with the denture to prevent causing an additional resistance against the denture's removal by touching the secondary crown.

Another aspect is which force at which point of time is the decisive factor for satisfying denture retention. Is the maximum force measured during the dentures removal decisive or is the value of the initial removal the more important value to be measured, as the denture should ideally stay absolutely in position during chewing? Anyhow, a high retention force value reached after $2 \mathrm{~mm}$ of the dentures removal could be combined with a great vertical resilience. This can cause reduced denture stability resulting in food impaction below the dentures base and less comfort for the patient. So, the initial retention force value seems to be the more relevant parameter for denture retention.

\section{Samples}

The samples used for measurement in this combined in vitro/ in vivo study should ideally have had parallel friction surfaces. Many laboratories tend to produce crowns with a slightly conical angle of $1-2^{\circ}$ to facilitate preparation during the milling process. It also has to be recommended that minimal conicity of the primary crowns can occur very easily, as milling absolutely parallel surfaces is extremely difficult. The surface of the occlusal half therefore tends to be prepared slightly more to avoid undercuts in the primary crown. The retention force of the double crown is influenced by the dental technician and is dependent on many factors. Milling speed, degree of wear of the cutters, polishing, casting technique, and method of setting the retention forces vary greatly and produce a very wide distribution of the values $[15,25]$. In addition, the random sample examined was nonhomogeneous with regard to the type of tooth. At prosthesis using an overdenture design, the possibility of an additional vacuum effect was avoided by venting the seal of the denture's flange. The tip of the measuring device was positioned carefully to avoid injuries of the gingival tissues.

Analysis of the results

In this study the initial median in vivo retention force value of an individual double crown reached $4.7 \mathrm{~N}$. These values are in good accordance to the measurements of in vitro studies discussing a retentive force of 4-9 $\mathrm{N}$ on different double crown techniques $[4,17,26]$. The retention force of double crowns depends on many different variables during the fabrication process. Laboratory tests have ultimately provided data recommending the withdrawal force for double crowns $[15$, 27]. These data were, however, based on measurements of samples, which were fabricated under ideal, standardized manufacturing conditions. In clinical practice the dental technician ultimately determines the retention force [28]. Only few data have been available up to now on the average clinical withdrawal forces of individual telescopic crowns. A previous study by Stancic and Jelenkovic also differentiated between the withdrawal forces of telescopic crowns on different types of tooth [4]. In good accordance to the present study, they determined $6.5 \mathrm{~N}$ for the specimens on canines and about $3 \mathrm{~N}$ for those on molars. This study measured the retentive forces of 20 individual dentures.

The development of the retentive forces within the period of 18 months showed a slight decrease of the values. The decrease was statistically significant. A retention force decrease was also documented by different in vitro studies of retentive elements [3, 15, 29]. For the clinically found retention force decrease of telescopic crowns, there are only a few data. Even though these findings were also documented for telescopic crowns $[8,9]$, a measurement is needed to analyze the long-term retention force development. As result only studies about magnets, bars and ball anchors can be used for the comparison of the values [21-24, 30]. These studies always measured the retentive force for the denture and not the force related to the single attachment. As problem there exists, no measuring system allowing a precise measurement of double crown retained dentures in an ideally way. Even little divergences of the crowns' axes and the direction of removal create a tipping of the double crowns, as the denture's framework connects the secondary crowns. This tilting of the double system raises the retention force enormously. To avoid this problem, only the initial removal was performed. Additionally, this situation equates to the clinically relevant situation of a one-sided chewing of sticky food. This chewing also results in one-sided removal forces. To achieve a value for the comparison with the recent literature, the summation of the single measurement points was performed. The results showed that the force of the 
double dentures does not change significantly within the period of 18 months. The range of the present median values $(11-13 \mathrm{~N})$ was in good accordance to the results of the recent literature that showed about 4-16 $\mathrm{N}$ per denture [21-24, 30].

\section{Conclusion}

The results of this study allow the following conclusions to be drawn regarding the withdrawal force of double crowns in clinical practice:

1. The retention force of dentures retained by doublecrown systems does not change clinically relevant within the first 1.5 years.

2. The retentive force values reach the same range as the values measured for other retentive systems.

3. After 18 months of intraoral usage, the retention force seems to be still sufficient for denture retention.

The question is how much overall retention force is necessary to produce a denture that is sufficiently stable, functional, and satisfactory for the patient. Ultimately, this question can only be answered by further investigation to clarify whether the retention force of the dentures can be correlated with the patient's satisfaction.

Conflict of interest The authors declare that they have no conflict of interest.

\section{References}

1. Becerra G, MacEntee M (1987) A classification of precision attachments. J Prosthet Dent 58:322-327

2. Wolfe RE (1985) Symposium on semiprecision attachments in removable partial dentures. Extracoronal attachments. Dent Clin North Am 29:185-198

3. Bayer S, Steinheuser D, Gruner M, Keilig L, Enkling N, Stark H, Mues S (2009) Comparative study of four retentive anchor systems for implant supported overdentures - retention force changes. Gerodontology 26:268-272

4. Stancic I, Jelenkovic A (2008) Retention of telescopic denture in elderly patients with maximum partially edentulous arch. Gerodontology 25:162-167

5. Wostmann B, Balkenhol M, Kothe A, Ferger P (2008) Dental impact on daily living of telescopic crown-retained partial dentures. Int J Prosthodont 21:419-421

6. Behr M, Hofmann E, Rosentritt M, Lang R, Handel G (2000) Technical failure rates of double crown-retained removable partial dentures. Clin Oral Investig 4:87-90

7. Wostmann B, Balkenhol M, Weber A, Ferger P, Rehmann P (2007) Long-term analysis of telescopic crown retained removable partial dentures: survival and need for maintenance. J Dent 35:939-945

8. Stark H, Schrenker H (1998) Telescopic crown retained dentures - a clinical long-term study. Dtsch Zahnärztl Z 3:183-186
9. Stark H (1996) Clinical and material studies of telescopic dentures - clinical long-term results and wear tests. HänselHohenhausen, Frankfurt

10. Igarashi Y, Goto T (1997) Ten-year follow-up study of conical crown-retained dentures. Int J Prosthodont 10(2):149-155

11. Beschnidt SM, Chitmongkolsuk S, Prull R (2001) Telescopic crown-retained removable partial dentures: review and case report. Compend Contin Educ Dent 22(11):927-928

12. Bergman B, Ericson A, Molin M (1996) Long-term clinical results after treatment with conical crown-retained dentures. Int $\mathrm{J}$ Prosthodont 9(6):533-538

13. Grossmann AC, Hassel AJ, Schilling O, Lehmann F, Koob A, Rammelsberg P (2007) Treatment with double crown-retained removable partial dentures and oral health-related quality of life in middle- and high-aged patients. Int J Prosthodont 20(6):576-578

14. Mock F, Schrenker H, Stark HK (2005) Success of telescopic crowns - a prospective long term study. Dtsch Zahnärztl Z 3 (60):148-153

15. Hagner MWHR, Grüner M, Bayer S, Keilig L, Reimann S, Bourauel C, Utz KH, Stark H (2006) Wear analysis of telescopic crowns - an in vitro study. Dtsch Zahnärztl Z 11(61):594-603

16. Becker H (1983) Retention mechanism of telescopic crowns. Zahnarztl Prax 34(7):281-284

17. Korber KH (1983) Conical crowns - a rational telescopic system. ZWR 92(2):38-43

18. Botega DM, Mesquita MF, Henriques GE, Vaz LG (2004) Retention force and fatigue strength of overdenture attachment systems. J Oral Rehabil 31(9):884-889

19. Lehmann KM, Armin FV (1976) Study of retentive forces of ball anchors. Schweiz Monatsschr Zahnmed 86:521-530

20. Bayer S, Stark H, Mues S, Keilig L, Schrader A, Enkling N (2009) Retention force measurement of telescopic crowns. Clin Oral Investig 14(5):607-611

21. Naert I, Gizani S, Vuylsteke M, Van Steenberghe D (1999) A 5-year prospective randomized clinical trial on the influence of splinted and unsplinted oral implants retaining a mandibular overdenture: prosthetic aspects and patient satisfaction. J Oral Rehabil 26 (3):195-202

22. van Kampen F, Cune M, van der Bilt A, Bosman F (2003) Retention and postinsertion maintenance of bar-clip, ball and magnet attachments in mandibular implant overdenture treatment: an in vivo comparison after 3 months of function. Clin Oral Implants Res 14(6):720-726

23. Burns DR, Unger JW, Elswick RK Jr, Beck DA (1995) Prospective clinical evaluation of mandibular implant overdentures: Part IRetention, stability, and tissue response. J Prosthet Dent 73(4):354-363

24. Naert I, Quirynen M, Hooghe M, van Steenberghe D (1994) A comparative prospective study of splinted and unsplinted Branemark implants in mandibular overdenture therapy: a preliminary report. J Prosthet Dent 71(5):486-492

25. Stuttgen U (1983) Experimental study of the parallelism of milled telescoping primary anchors. Dtsch Zahnärztl Z 38(5):538-540

26. Minagi S, Natsuaki N, Nishigawa G, Sato T (1999) New telescopic crown design for removable partial dentures. J Prosthet Dent 81(6):684-688

27. Gungor MA, Artunc C, Sonugelen M (2004) Parameters affecting retentive force of conus crowns. J Oral Rehabil 31(3):271-277

28. Bohr M (2000) Clinical examination of a technique for retention force adjustment of telescopic crowns. Dissertation, University of Hamburg

29. Holst S, Blatz MB, Eitner S, Wichmann M (2006) In vitro wear of different material combinations of intracoronal precision attachments. Int J Prosthodont 19(4):330-332

30. Naert IE, Gizani S, Vuylsteke M, van Steenberghe D (1997) A randomised clinical trial on the influence of splinted and unsplinted oral implants in mandibular overdenture therapy. A 3-year report. Clin Oral Investig 1(2):81-88 